\title{
Sound power estimation by laser Doppler vibration measurement techniques
}

\author{
G.M. Revel * and G.L. Rossi \\ Dipartimento di Meccanica, Università degli Studi di \\ Ancona, I-60131 Ancona, Italy
}

Received 19 December 1997

Revised 21 September 1998

The aim of this paper is to propose simple and quick methods for the determination of the sound power emitted by a vibrating surface, by using non-contact vibration measurement techniques. In order to calculate the acoustic power by vibration data processing, two different approaches are presented. The first is based on the method proposed in the Standard ISO/TR 7849, while the second is based on the superposition theorem. A laser-Doppler scanning vibrometer has been employed for vibration measurements. Laser techniques open up new possibilities in this field because of their high spatial resolution and their non-intrusivity. The technique has been applied here to estimate the acoustic power emitted by a loudspeaker diaphragm. Results have been compared with those from a commercial Boundary Element Method (BEM) software and experimentally validated by acoustic intensity measurements. Predicted and experimental results seem to be in agreement (differences lower than $1 \mathrm{~dB}$ ) thus showing that the proposed techniques can be employed as rapid solutions for many practical and industrial applications. Uncertainty sources are addressed and their effect is discussed.

\section{Introduction}

In many problems of acoustics, sound is generated by solid vibrating surfaces of structures or machines which are within environments where several noise sources are present simultaneously (e.g., production lines). The acoustic field is then related to all the sources, and it can be difficult and time consuming, either by intensity measurement systems [7] or by acous-

\footnotetext{
${ }^{*}$ Corresponding author: Dr. Gian Marco Revel, Dipartimento di Meccanica, Università degli Studi di Ancona, Via Brecce Bianche, I-60131 Ancona, Italy, Tel.: +39 71 2204441; Fax: +39 71 2204801; E-mail: revel@mehp1.cineca.it.
}

tic array techniques (based on spatial transformations [14] or IFRF methods [6]), to detect the contribution to the noise from each particular surface. In these cases two typical questions are of technical interest:

1) What is the contribution of a particular surface to total emitted sound power?

2) Where is the surface emitting a particular frequency of noise?

The first question is important when the emission of a particular machine or structure component must be evaluated (e.g., in order to choose between different solutions for noise source reduction). The second question is of interest to avoid, for example, tedious tones in the noise spectrum emitted by a machine, in which case the source is usually identified using vibration measurements.

Since the spatial resolution of acoustic intensity measurements or array techniques depends on the sound wavelength, in some applications where small size sources are involved, it can be difficult to give a clear experimental answer to the above questions by these measurement techniques. These are typical cases where vibration measurements are necessary to better identify and characterise noise sources: in fact, the acoustic field can be calculated by processing the vibratory velocity data measured on the source surface $[2,10,13]$.

A laser Doppler scanning vibrometer [5] allows to obtain information on the vibration velocity of a surface with very high spatial resolution and in a large number of points. A laser beam is focused on the measurement point and the instantaneous velocity of the surface in the laser beam direction is determined using the Doppler effect induced on the scattered laser light. This instrument can therefore be used to solve, experimentally, acoustic problems like those mentioned above. Furthermore, because of its non-intrusivity, it can be employed in applications where non contact measurement devices are needed, such as on very light structures. 


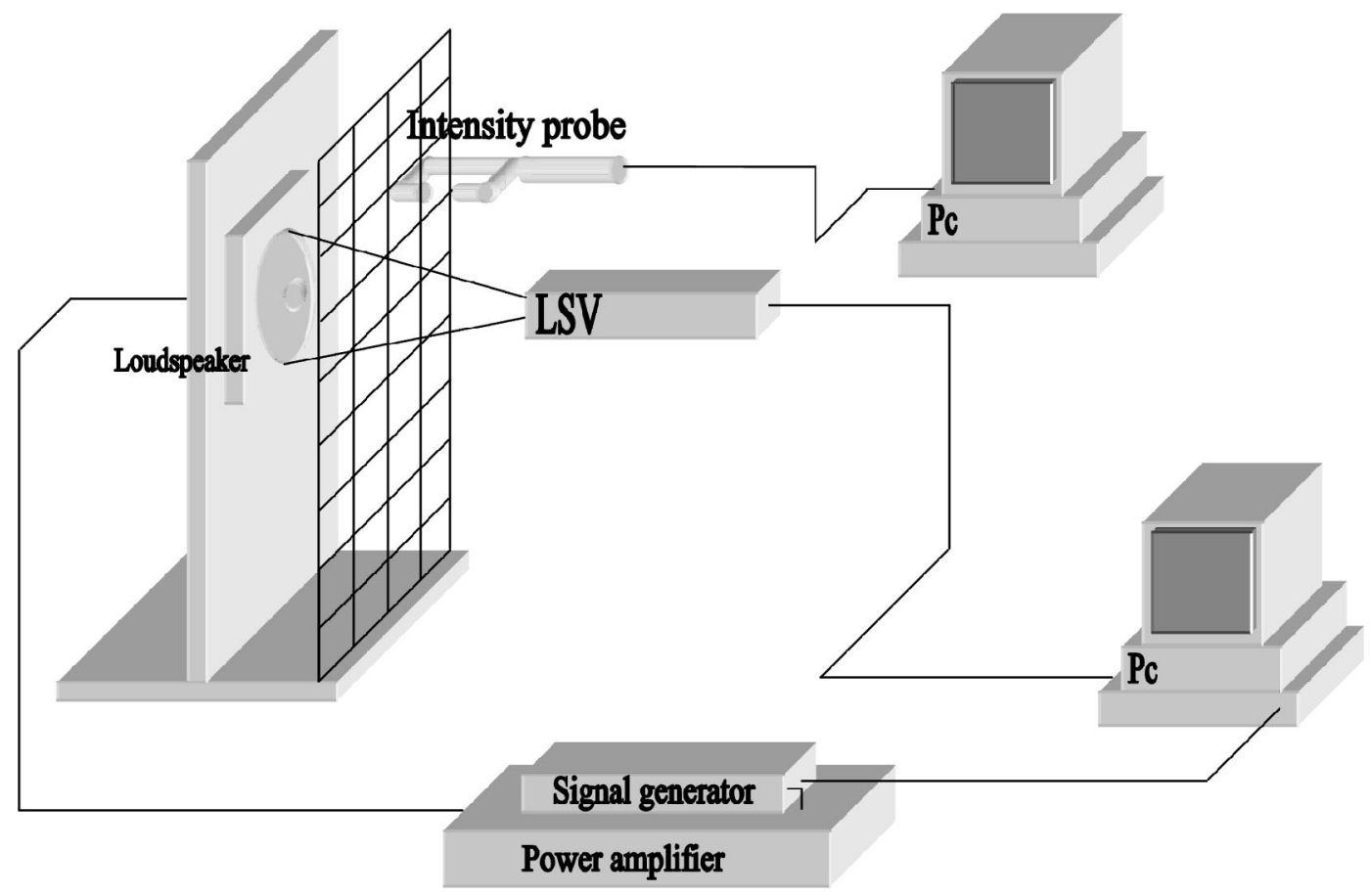

Fig. 1. Experimental set-up for vibration and acoustic intensity measurements.

In previous works $[11,12]$ the authors have proposed the use of laser scanning vibrometers to get experimental vibration data to employ as boundary conditions for acoustic Boundary Element Method (BEM) [2] codes. These are very robust tools for acoustic field prediction, but commercial BEM software are expensive and require very powerful computers and long processing time.

An ISO (International Standard Organization) Technical Report exists [9] that suggests a technique to estimate acoustic power emission based on vibration measurements taken on a vibrating surface. Until now this Technical Report has not been frequently used because of the lack of techniques allowing high spatial resolution measurements of surface vibration. Therefore, the first idea developed in this work is the employment of laser scanning vibrometers to provide experimental data for the ISO data processing algorithm.

Given that phase information can also be achieved by vibration measurements, another approach has been developed in order to calculate noise emission using vibration data. In this approach, the superposition theorem and simple acoustic relationships $[10,13]$ have been applied to compute the pressure on a point at a certain distance from the source (taking into account the contribution from all the points of the sur- face). Then the sound power flowing through a desired area has been determined. A similar approach (but with different formulation) has been previously reported [17] that calculates the acoustic emission of a vibrating plate using accelerometers to measure vibrations. Another time domain processing technique has also been proposed, with the same purposes, for impulsive noise [8].

These methods present many advantages over microphone based techniques, particularly in terms of infield applicability, since other noise sources often have negligible effects on the vibration of the emitting surface.

\section{Case study: a loudspeaker diaphragm}

In order to achieve an experimental validation of the proposed technique for sound power measurement, the surface of a loudspeaker diaphragm has been used as noise source. This particular vibrating structure has been chosen because of its interesting behaviour: its vibration is similar to a "rigid piston" at low frequencies and it exhibits a combination of vibration modes of the diaphragm at medium or high frequencies $[1,3]$. These features make it suitable to characterise the pro- 


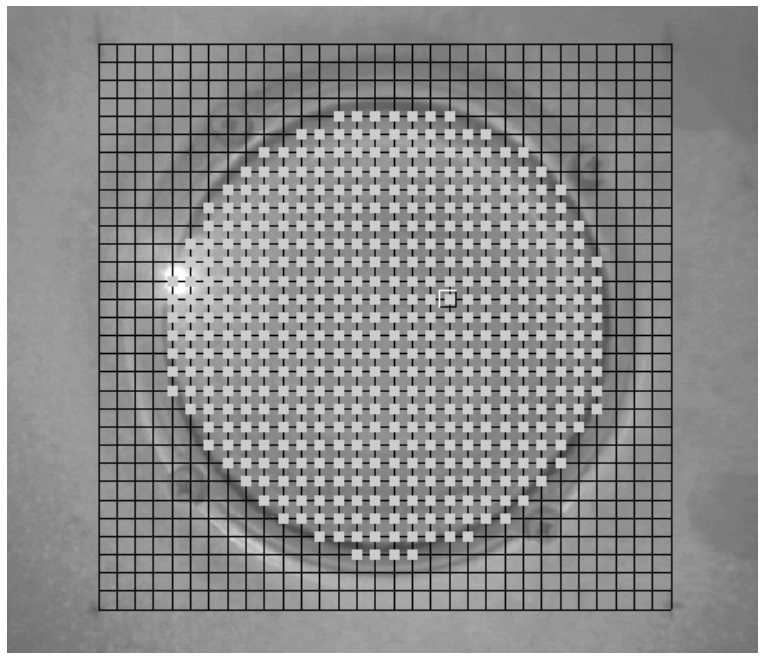

Fig. 2. The grid used for vibration measurements on the loudspeaker diaphragm (image from the vibrometer head camera).

cedure both in simple conditions, which are typical for preliminary calibration of new methods, and in more complex conditions, similar to those of real applications.

The loudspeaker used is a subwoofer with a flat diaphragm (105 mm diameter) which, from preliminary tests, has shown a behaviour similar to that of a rigid piston up to $400 \mathrm{~Hz}$, while, at higher frequencies, vibration modes with reduction of the area of the moving surface appear [15]. Vibration measurement on a loudspeaker is one of the typical cases where, because of the diaphragm lightness, non contacting techniques are required.

The excitation frequencies chosen for tests were $300 \mathrm{~Hz}$ and $1500 \mathrm{~Hz}$. The loudspeaker was mounted on a rigid wood panel ( $20 \mathrm{~mm}$ thick) of $1 \mathrm{~m}$ length and $1.5 \mathrm{~m}$ height (Fig. 1). A sinusoidal excitation has been used with a amplitude of $8 \mathrm{~V}$ RMS.

Vibration measurements on the loudspeaker diaphragm surface have been performed on a grid of 463 points (Fig. 2) using a Polytec PSV 200 laser Doppler scanning vibrometer. The output signal of the signal generator has also been acquired in order to have a phase reference. Vibration magnitude, measured at $300 \mathrm{~Hz}$ and $1500 \mathrm{~Hz}$, are reported in Figs 3 and 4 , respectively. A symmetric "piston like" motion is evident at $300 \mathrm{~Hz}$ (with same phase at all the measured points), while a complex mode shape appears at $1500 \mathrm{~Hz}$.

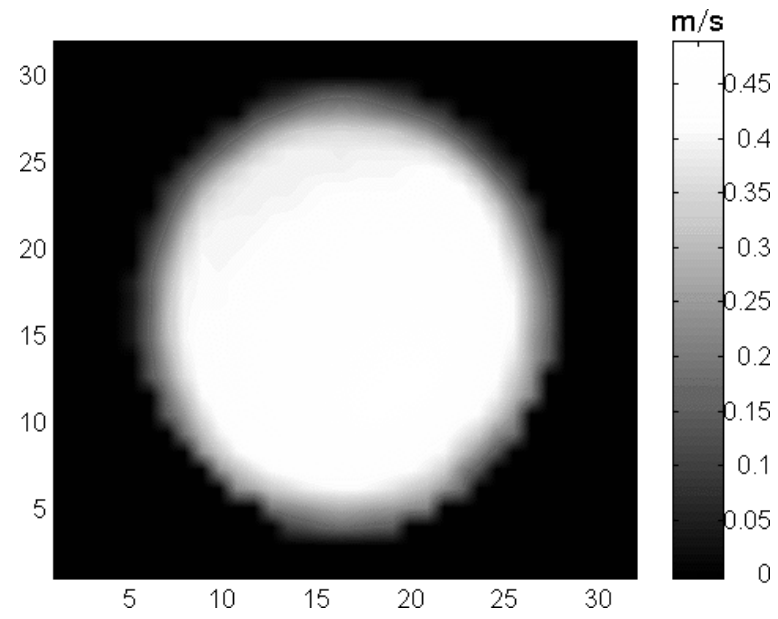

Fig. 3. Vibration velocity magnitude distribution at $300 \mathrm{~Hz}$.

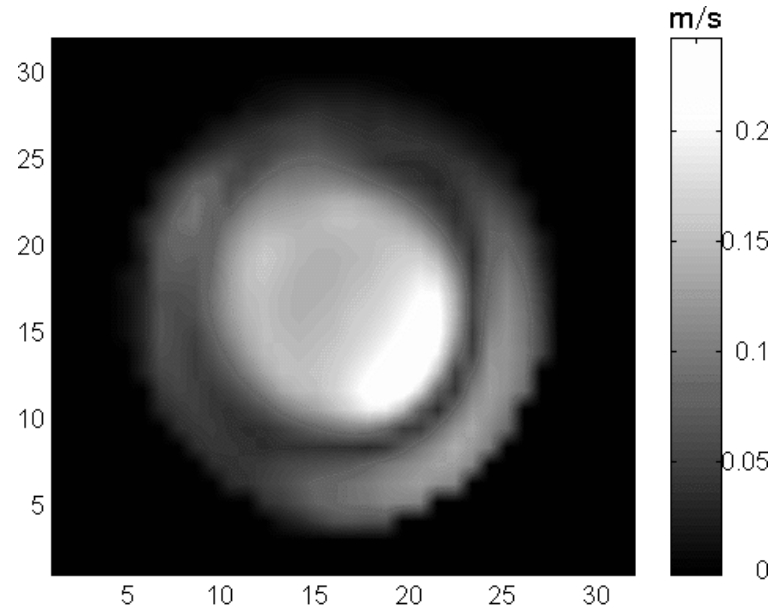

Fig. 4. Vibration velocity magnitude distribution at $1500 \mathrm{~Hz}$.

\section{The proposed methods for vibration data processing}

\subsection{The ISO/TR 7849 method}

The first algorithm proposed is that of the ISO/TR 7849 Technical Report [9] where the total airborne sound power $W_{\text {tot }}$ emitted by a surface of area $S$ is estimated according to the following equation:

$$
W_{\mathrm{tot}}=\sigma \rho c S \overline{v^{2}}
$$

where $\rho$ is the mean air density, $c$ is the sound velocity in air, $v^{2}$ is the mean square value of the normal velocity averaged over the surface area $S$ and $\sigma$ is the radia- 
Table 1

Sound power values estimated using the different methods

\begin{tabular}{ccccc}
\hline Frequency & $\begin{array}{c}\text { ISO/TR 7849 } \\
\text { method }\end{array}$ & $\begin{array}{c}\text { Superposition } \\
\text { method }\end{array}$ & $\begin{array}{c}\text { Boundary element } \\
\text { method }\end{array}$ & $\begin{array}{c}\text { Intensity } \\
\text { measurement }\end{array}$ \\
\hline $300 \mathrm{~Hz}$ & $99.6 \mathrm{~dB}$ & $98.4 \mathrm{~dB}$ & $97.2 \mathrm{~dB}$ & $98.1 \mathrm{~dB}$ \\
$1500 \mathrm{~Hz}$ & $100.5 \mathrm{~dB}$ & $99 \mathrm{~dB}$ & $96 \mathrm{~dB}$ & $99.2 \mathrm{~dB}$ \\
\hline
\end{tabular}

tion factor, which is described (Eq. 2) as a function of the sound frequency $f(\mathrm{~Hz})$ and of the source typical dimension $R$ (the loudspeaker diaphragm radius in the case study):

$$
10 \log \sigma=-\log \left[1+0.1 \frac{c^{2}}{(f R)^{2}}\right] .
$$

A limit of this method is that it does not supply information about the spatial distribution of the acoustic field. Furthermore, in some particular applications the accuracy of the method has been considered too poor and so, when it was proposed in 1982, it was decided that it was not yet suitable for an International Standard, but only for a Technical Report. Now, the recent developments of laser techniques open up new possibilities to its application, as an increase of the vibration measurement spatial resolution reduces significantly the uncertainty in the estimation of the mean square value of the vibratory velocity over the surface.

Using a procedure implemented in Matlab ${ }^{\circledR}$, the sound power values shown in Table 1 have been found.

\subsection{The method based on the linear superposition of effects}

In the second method both phase information and spatial distribution of the vibration data have been considered, in such a way as to predict the acoustic field in front of the loudspeaker.

Since the aim of the work is to implement a simple tool for sound power measurement based on laser vibrometer data, the elementary model of radiation from a circular piston of area $S$, mounted flush with the surface of an infinite baffle and vibrating with simple harmonic motion, has been employed. This assumption can be considered correct for the frequencies investigated, since the panel dimension is larger then half of the sound wavelength $\lambda$.

In the developed model we assume that the piston is composed of an array of simple sources $A_{i}$, each corresponding to the portion of area with centre in the vibration measurement point. In the experiments, where a grid of 463 points has been used, each element $A_{i}$ has an emitting surface $S_{i}$ (where $\sum_{i} S_{i}=S$ ) equal to a square of $4.4 \mathrm{~mm}$ side (Fig. 2). The laser vibrometer allows to resolve the vibration mode of the surface quickly and with high spatial resolution, this being necessary to describe the acoustic field with accuracy, particularly when velocity and phase of different points vary significantly.

The acoustic pressure $p_{i h}$ radiated by the $i$-th simple source $A_{i}$ at a distance $r_{i h}$ (Fig. 5) in a point $P_{h}$ of the space in front of the source is $[10,13]$ :

$$
p_{i h}=S_{i} \frac{\mathrm{j} \rho c k v_{i}}{2 \pi r_{i h}} \mathrm{e}^{\mathrm{j}\left(\omega t-k r_{i h}-\phi_{i}\right)},
$$

where $\mathrm{j}(=\sqrt{-1})$ is the complex value, $\omega=2 \pi f$ is angular frequency $(\mathrm{rad} / \mathrm{s}), k=\omega / c$ is the wave number, $v_{i}$ and $\phi_{i}$ are the vibration velocity magnitude and phase measured in $A_{i}$.

The total sound pressure $p_{h}$ at $P_{h}$, due to the whole diaphragm vibration, can be estimated by means of the linear superposition of effects, i.e., by summing the contributions of all the elements $A_{i}(i=1, \ldots, n$, where $n$ is the number of vibration measurement points, $n=463$ in this case) of the source:

$$
p_{h}=\frac{\mathrm{j} \rho c k}{2 \pi} \sum_{i=1}^{n} S_{i} \frac{v_{i}}{r_{i h}} \mathrm{e}^{\mathrm{j}\left(\omega t-k r_{i h}-\phi_{i}\right)}
$$

Then the RMS value of $p_{h}$ in a time interval $T$ is calculated as follows:

$$
\begin{aligned}
p_{h, \mathrm{rms}} & =\sqrt{\lim _{T \rightarrow \infty} \frac{1}{T} \int_{0}^{T} p_{h}^{2} \mathrm{~d} t} \\
& =\sqrt{\frac{1}{2} \sum_{i=1}^{n} \sum_{k=1}^{n} B_{i h} B_{k h} \cos \left(\Phi_{i h}-\Phi_{k h}\right)},
\end{aligned}
$$

where:

$$
\begin{aligned}
B_{i h} & =\frac{\rho c k v_{i}}{2 \pi r_{i h}} S_{i}, \\
\Phi_{i h}-\Phi_{k h} & =\left(k r_{i h}+\phi_{i}\right)-\left(k r_{k h}+\phi_{k}\right) \\
& =k\left(r_{i h}-r_{k h}\right)+\left(\phi_{i}-\phi_{k}\right) .
\end{aligned}
$$




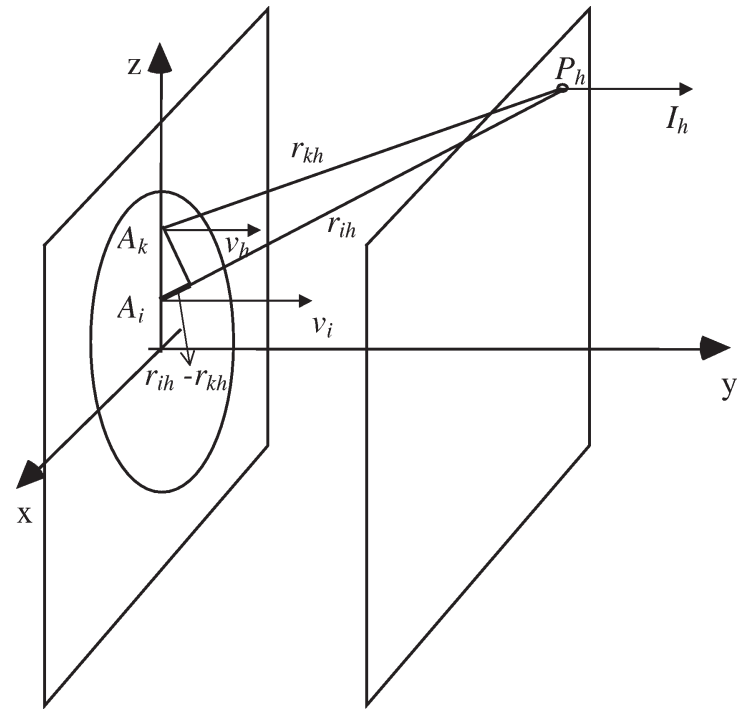

Fig. 5. Scheme used for sound power estimation.

Therefore, in order to describe the directivity of the source, for the contributions of each couple of points ( $A_{i}$ and $A_{k}$, Fig. 5) of the measurement grid, the phase difference has to be considered, which has two components. The first is due to the difference of path length between each simple source and $P_{h}\left(r_{i h}-r_{k h}\right)$ and depends on geometry; the second is due to the different initial phases $\left(\phi_{i}-\phi_{k}\right)$. A laser scanning vibrometer allows to measure phase differences if a reference signal is used. Here the driving signal from the generator has been used as reference, but in other applications an accelerometer, a single-point laser vibrometer or a microphone will be well suited.

The total acoustic intensity $I_{h}$ at point $P_{h}$ is given by:

$$
I_{h}=\frac{p_{h, \mathrm{rms}}^{2}}{\rho c} .
$$

Results obtained on a grid of total area $Z=(1 \mathrm{~m} \times$ $1 \mathrm{~m}$ ) distant $0.5 \mathrm{~m}$ from the baffle panel surface are reported in Figs 6 and 7 at $300 \mathrm{~Hz}$ and $1500 \mathrm{~Hz}$, respectively; the grid is composed of $12 \times 12$ elements of area $Z_{h}$ (where $\sum_{h} Z_{h}=Z$ ) and centre in $P_{h}(h=1, \ldots, m$ with $m=144$ in this case $)$. A circular intensity distribution has been obtained at $300 \mathrm{~Hz}$ (when the loudspeaker moves as a rigid piston), while an elliptic distribution with a sloping axis of symmetry, similar to that of the measured vibration pattern, is present at $1500 \mathrm{~Hz}$.

Finally, the total acoustic power $W_{Z \text {,tot }}$ flowing through the considered grid has been estimated as:

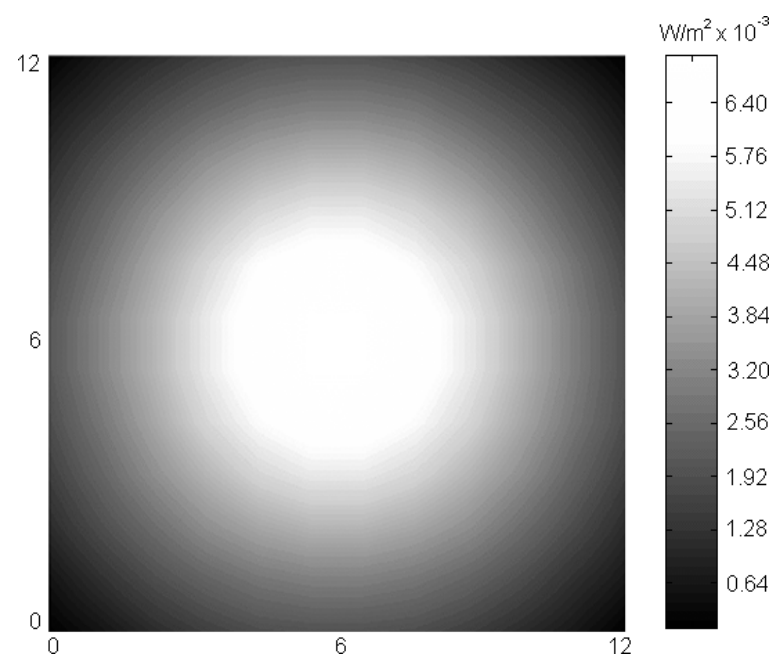

Fig. 6. Predicted acoustic intensity distribution at $300 \mathrm{~Hz}$.

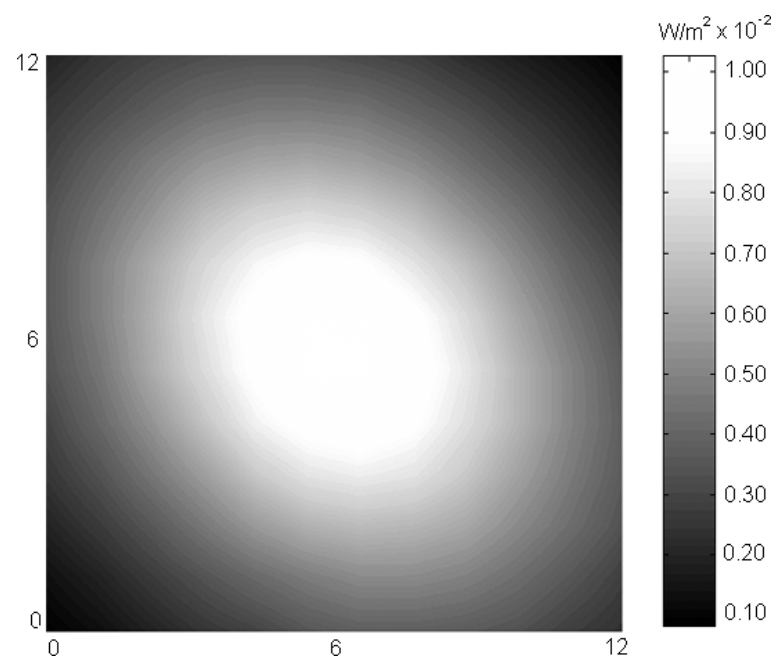

Fig. 7. Predicted acoustic intensity distribution at $1500 \mathrm{~Hz}$.

$$
W_{Z \text {,tot }}=\sum_{h=1}^{m} I_{h} Z_{h} .
$$

Results obtained, using a routine implemented in Matlab $^{\circledR}$, are shown in Table 1.

\section{Comparison with boundary element method results}

The prediction of the acoustic power emitted by the loudspeaker has been approached also by using a boundary element model implemented in a commercial code (SYSNOISE). BEM codes are actually becoming 

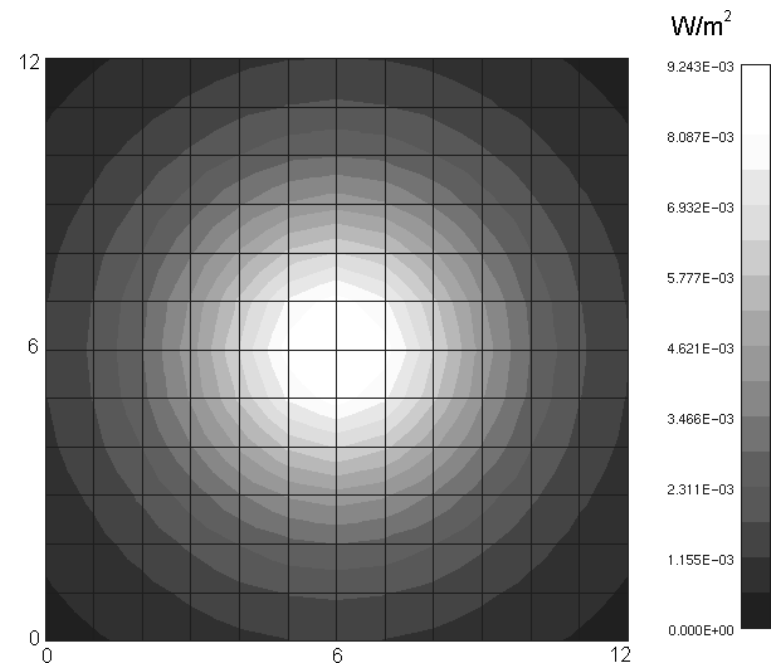

Fig. 8. Acoustic intensity distribution at $300 \mathrm{~Hz}$ computed by BEM.

a "standard" in the field of acoustic prediction, because of their large potential: they are able to import data and complex geometry from finite element models (which can be used for prediction of the dynamic behaviour of the structure), to solve both interior and exterior acoustic problems, also considering scattering phenomena, and to present results in many different graphic ways. The main problems that hinder the widespread use of BEM codes are connected to the fact that they are expensive and require very powerful computers and long processing times.

It is well known that in the frequency domain the pressure $p_{h}$ at any point $P_{h}$ has to satisfy the Helmholtz equation $[10,13]$ :

$$
\nabla^{2} p_{h}(\omega)+k^{2} p_{h}(\omega)=0,
$$

where $\nabla^{2}$ is the Laplacian operator.

In the BEM formulation [2,16], the acoustic problem is solved by applying the Green's third identity to achieve an integral form of the Helmholtz equation as follows:

$$
\begin{aligned}
& \int_{S}\left[p_{i}(\omega) \frac{\mathrm{d}}{\mathrm{d} \eta}\left(G_{i h}\right)-G_{i h} \frac{\mathrm{d}}{\mathrm{d} \eta}\left(p_{i}(\omega)\right)\right] \mathrm{d} S_{i} \\
& \quad=c_{h} p_{h}(\omega),
\end{aligned}
$$

where $\mathrm{d} / \mathrm{d} \eta$ is the gradient operator on the surface normal $\eta, G_{i h}$ is the Green's function, which is given by:

$$
G_{i h}=\frac{\mathrm{e}^{-\mathrm{j} k r_{i h}}}{4 \pi r_{i h}}
$$
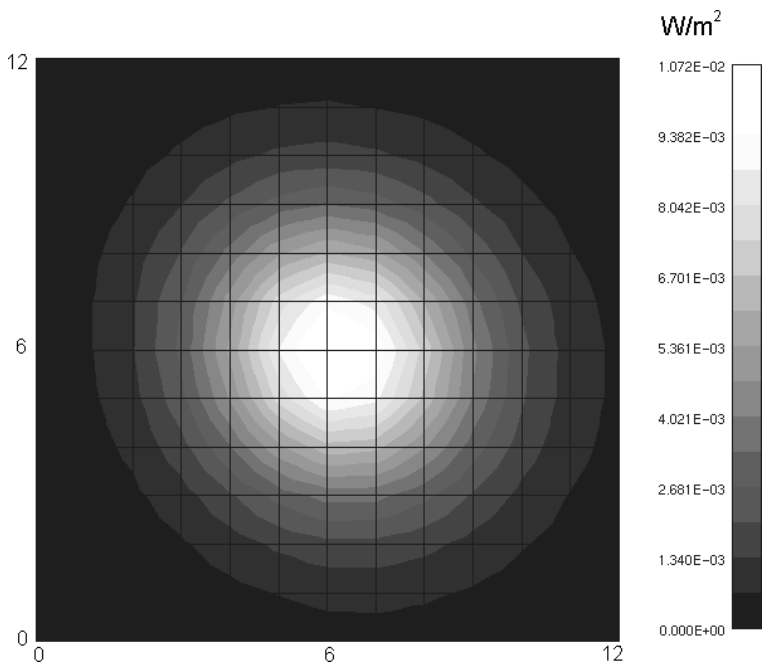

Fig. 9. Acoustic intensity distribution at $1500 \mathrm{~Hz}$ computed by BEM.

and $c_{h}$, the so called Helmholtz constant, is 1 for a point $P_{h}$ in the acoustic field and $1 / 2$ for a boundary element $A_{i}$ on the vibrating surface.

The Helmholtz integral equation (Eq. (10)) can be solved for the boundary elements on the surface by imposing the boundary conditions, which can take the form of prescribed velocity, pressure or admittance. Once known the pressure $p_{i}$ and its derivatives at the surface point $A_{i}$, the acoustic variables will be predicted by Eq. (10) at any point $P_{h}$.

In the case studied, the loudspeaker has been modelled as a piston mounted on an infinite rigid baffle and the velocity components $v_{i}$, measured by the laser Doppler vibrometer, have been employed as boundary conditions of the problem (Neumann boundary condition [16]), applied in the following form:

$$
\frac{\mathrm{d}}{\mathrm{d} \eta}\left(p_{i}(\omega)\right)=-\mathrm{j} \rho \omega v_{i}(\omega)
$$

In Figs 8 and 9 the acoustic intensity distributions at 300 and $1500 \mathrm{~Hz}$ are shown. As expected they correlate well with those previously computed (Figs 6 and 7); in particular, at $1500 \mathrm{~Hz}$ the same sloping axis of symmetry of Fig. 7 is present.

For the total sound power, the results found are shown in Table 1.

\section{Comparison with acoustic intensity measurements}

Finally, results predicted by the different methods have been compared with experimental results 


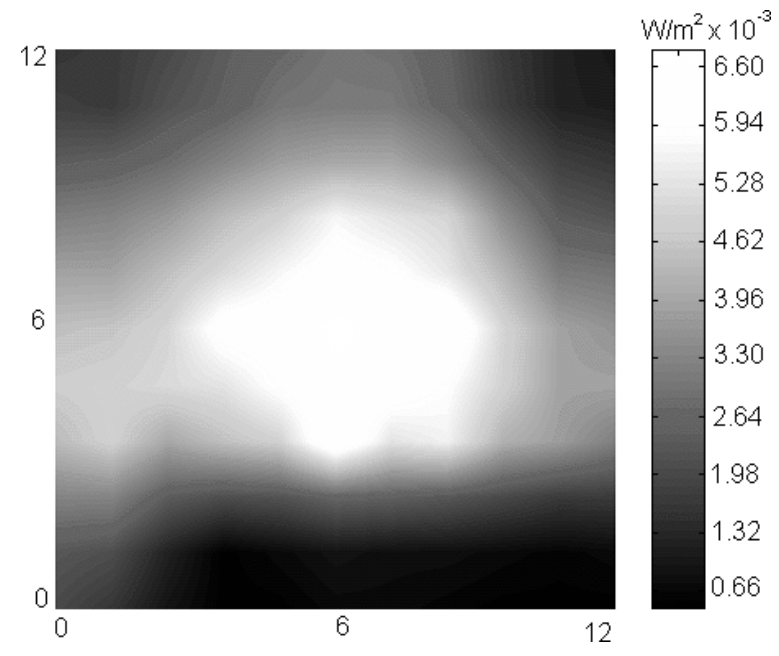

Fig. 10. Measured acoustic intensity distribution at $300 \mathrm{~Hz}$.

obtained using the acoustic intensity technique [7]. Acoustic intensity measurements have been performed on a grid distant $0.5 \mathrm{~m}$ from the panel (Fig. 1). The dimensions of the grid were $1 \mathrm{~m} \times 1 \mathrm{~m}$ and the spatial resolution was $12 \times 12$ measurement points.

Measurements have been performed using a Bruel and Kjaer (B\&K) 3548 two-microphone intensity probe, calibrated by a B\&K 3541 calibrator and then connected with a B\&K 2144 spectrum analyser. For the system control, data acquisition and processing, the B\&K Noise Source Location and Sound Power software were employed. Accordingly to the ISO 97141 standard, measurement parameters have been set in such a way as to have results classified at "precision grade". For this kind of measurement, ISO 9614-1 standard states a repeatability (between different laboratories) of $1.5 \mathrm{~dB}$ at $300 \mathrm{~Hz}$ and of $1.0 \mathrm{~dB}$ at $1500 \mathrm{~Hz}$. Repeated measurements and calibrations and estimation of possible interfering inputs have allowed to consider those values as an overestimate of the uncertainty of the experimental results here presented.

The total sound power values achieved from the measurements are shown in Table 1.

In order to characterise the background noise of the test room (whose dimensions are $9 \times 13.5 \times 3.5 \mathrm{~m}$ ), repeated acoustic intensity measurements have been performed on the same grid with the loudspeaker switched off. A total sound power of $32 \pm 5 \mathrm{~dB}$ was obtained.

The measured intensity distributions are shown in Figs 10 and 11: they seem to be in good agreement with those calculated from vibration measurement results, in particular for what concerns their symmetry.

Differences are mainly due to the hypothesis of free field conditions used for calculations: in the distribu-

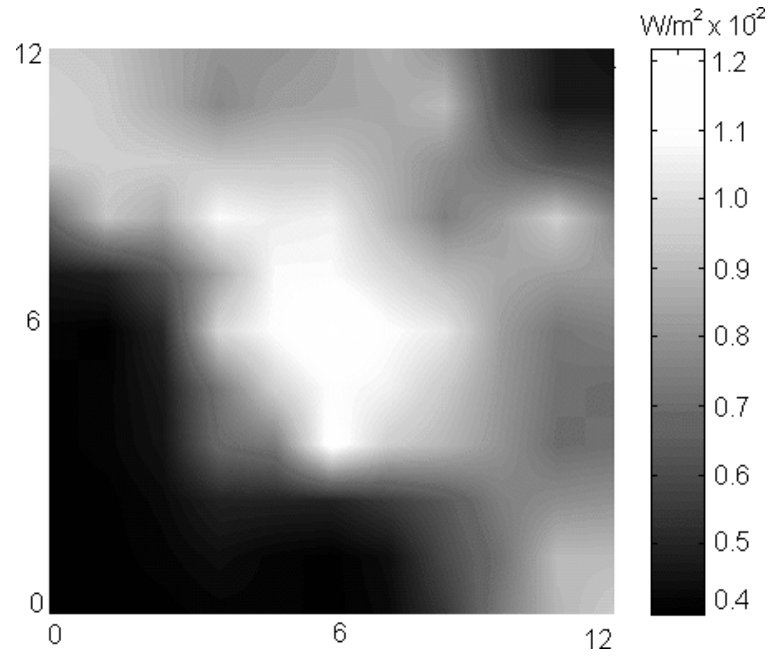

Fig. 11. Measured acoustic intensity distribution at $1500 \mathrm{~Hz}$.

tion of the measured maps the effects of the ground and of the panel are evident, which were not considered in the processing of vibration data. This is not a source of uncertainty, since the proposed methods aim to develop a tool for the measurement of the sound power emitted from a particular surface, not to estimate the acoustic field in reverberating conditions.

Effective sources of uncertainty for the method based on the superposition of effects are those arising from experimental vibration measurements, velocity amplitude $v_{i}$ and phase $\phi_{i}$. In particular, phase uncertainty has to be considered carefully because of its nonlinear relationship with the total sound power. Also uncertainty on the determination of the geometric parameters (values of the position vector $r_{i h}$ and surfaces $S_{i}$ ) affects the results, but in first approximation they can be neglected. Thus, error propagation [4] in pressure estimation can be analysed by applying the root-sum square formula of uncertainty to Eqs (5) and (6) as follows:

$$
\begin{aligned}
\Delta p_{h, \mathrm{rms}}= & {\left[\sum_{i=1}^{n}\left(\frac{\partial p_{h, \mathrm{rms}}}{\partial v_{i}} \Delta v\right)^{2}\right.} \\
& \left.+\sum_{i=1}^{n}\left(\frac{\partial p_{h, \mathrm{rms}}}{\partial \phi_{i}} \Delta \phi\right)^{2}\right]^{1 / 2},
\end{aligned}
$$

where $\Delta p_{h, \mathrm{rms}}, \Delta v, \Delta \phi$ are the uncertainties on $p_{h, \mathrm{rms}}, v$ and $\phi$, respectively.

It is worth noting that Eq. (13) assumes a simple form in the case at $300 \mathrm{~Hz}$, when velocity and phase exhibit a constant distribution: 


$$
\Delta p_{h, \mathrm{rms}}=\frac{1}{\sqrt{2}} \frac{\rho c k S}{2 \pi r_{i h}} \Delta v
$$

In the calculation of Eq. (14), the values of $r_{i h}$ have been assumed to be the same for any element $A_{i}$. This approximation can be considered acceptable as long as the dimensions of the acoustic grid are larger than those of the source. In this case, uncertainty on pressure depends linearly on velocity uncertainty $\Delta v$, and not on the number of measurement points, $n$. In fact, the error component related to the spatial discretization is negligible, since the distributions can be considered constant. As the vibrometer is working in ideal conditions, the relative uncertainty on velocity can be assumed to be about $0.5 \%$. This causes the same relative uncertainty on the calculated pressure value and the double in the calculated total sound power (as the pressure value is squared to compute the intensity), which therefore can be estimated with a relative uncertainty of about $1 \%$ in the case at $300 \mathrm{~Hz}$.

In cases where velocity and phase distributions are not constant (as in the case at $1500 \mathrm{~Hz}$ ), the error component related to the spatial discretization is reduced if a large number of points are used for interpolation, while the component due to the uncertainty of the magnitude and phase measurements does not decrease. Because of that, the high spatial resolution of laser vibrometers plays a fundamental role in order to have low uncertainty on sound power estimation.

Another approximation here introduced concerns the measured velocity direction: a laser vibrometer measures the velocity in the direction of the laser beam, but the velocity component to be considered for acoustic emission is the one normal to the surface. In these experiments the facility has been set up in such a way as to have a negligible difference between these two directions. In fact, the distance between laser vibrometer and loudspeaker was about $3 \mathrm{~m}$. Considering that the loudspeaker has a diameter of about $105 \mathrm{~mm}$, the maximum measurement inclination angle was lower than $1^{\circ}$. Therefore, the resulting difference between normal and measured velocity was lower than $1 \%$. In other particular applications (e.g., when the source has a complex shape or when it has large dimensions with respect to its distance from the laser head) this must be taken into account dividing by the cosine of the measurement incidence angles.

\section{Conclusions}

In this paper two methods for sound power measurement have been presented, which are based on laser-
Doppler scanning vibrometer and post-processing of surface velocity data.

The techniques have been experimentally validated on a simple case study, a loudspeaker diaphragm. Results, compared with both those computed by a BEM code and those obtained by an intensity measurement system, have shown limited uncertainty (differences lower than $1 \mathrm{~dB}$ with respect to the sound intensity results). Also, measured and calculated spatial distributions of sound intensity correlate well. In particular, the method based on the ISO/TR 7849 Technical Report seems to be fast and easy to use, but it is unable to supply information about spatial distribution of the acoustic variables. On the other hand, the linear superposition of effects gives more complete results, but requires a larger number of calculations.

This work presented different approaches for sound power estimation. The two methods based on laser vibrometry can be used as a solution for many industrial and engineering problems (e.g., measurement of the sound power emitted by machines in a production line). They are quite simple to be implemented and, furthermore, in the literature the formulations for several types of sources are reported, which approximate well a large number of practical cases.

BEM codes constitute an upper end solution, since they can also be used for reverberating environments and complex shapes of the source, but they are expensive and require long processing times and powerful computers.

At the end we have the experimental acoustic intensity measurement techniques, which usually give the most reliable results, but in some cases (e.g., in the characterisation of small sources) present problems and uncertainty, especially for what concerns spatial resolution.

\section{References}

[1] I.J. Borwick, Loudspeaker and Headphone Handbook, Butterworth, London, 1988.

[2] R.D. Ciskowski and C.A. Brebbia, Boundary Element Methods in Acoustics, Computational Mechanics Publications and Elsevier Applied Science, Southampton, 1991.

[3] M. Colloms, High Performances Loudspeakers, Pentech, London, 1991.

[4] E.O. Doeblin, Measurement Systems: Application and Design, McGraw-Hill, Singapore, 1990.

[5] L.E. Drain, The Laser Doppler Technique, Wiley, New York, 1980 . 
[6] S.M. Dumbacher and D.L. Brown, Practical considerations of the IFRF technique as applied to noise path analysis and acoustical imaging, in: Proc. 15th International Modal Analysis Conference, Orlando, 1997, pp. 1677-1685.

[7] F.J. Fahy, Sound Intensity, Elsevier Applied Science, London, 1989.

[8] M. Goueygou, B. Piwakowsky and B. Delannoy, Time domain computing for predicting the acoustic field radiated by vibrating structures, in: Proc. Vibration and Noise'95, Venice, 1995, pp. 9-15.

[9] ISO/TR 7849, Estimation of airborne noise emitted by machinery using vibration measurement, 1987.

[10] L.E. Kinstler, A.R. Frey, A.B. Coppens and J.V. Sanders, Fundamentals of Acoustics, Wiley, New York, 1982.

[11] P. Mas, L. Cremers and P. Sas, The use of laser Doppler vibrometer measurements for the prediction of the internal sound pressure field, in: Proc. 14th International Modal Analysis Conference, Detroit, 1996, pp. 845-850.
[12] D.E. Montgomery, R.L. West, R.A. Burdisso and H.E. Camargo, Acoustic radiation prediction of a compressor from 3D experimental spatial dynamics modelling, in: Proc. 1st Int. Conf. Vibration Measurements by Laser Techniques, SPIE Vol. 2358, Ancona, Italy, 1994, pp. 281-291.

[13] A.D. Pierce, Acoustics, McGraw-Hill, New York, 1989.

[14] P. Rasmussen, Spatial transformation of sound fields, Sound and Vibration 29(5) (1995).

[15] G.L. Rossi and E.P. Tomasini, Vibration measurements of loudspeaker diaphragms by a laser scanning vibrometer, in: Proc. 13th Int. Modal Analysis Conference, Nashville, 1995, pp. 1205-1209.

[16] SYSNOISE User's Manual, Numerical Integration Technologies, Leuven (Belgium), 1992.

[17] G. Zhen, W. Zhongzhang and G. Tun, Relation between plate vibration and its sound field, J. Acoustic 32(1) (1989), 35-42. 

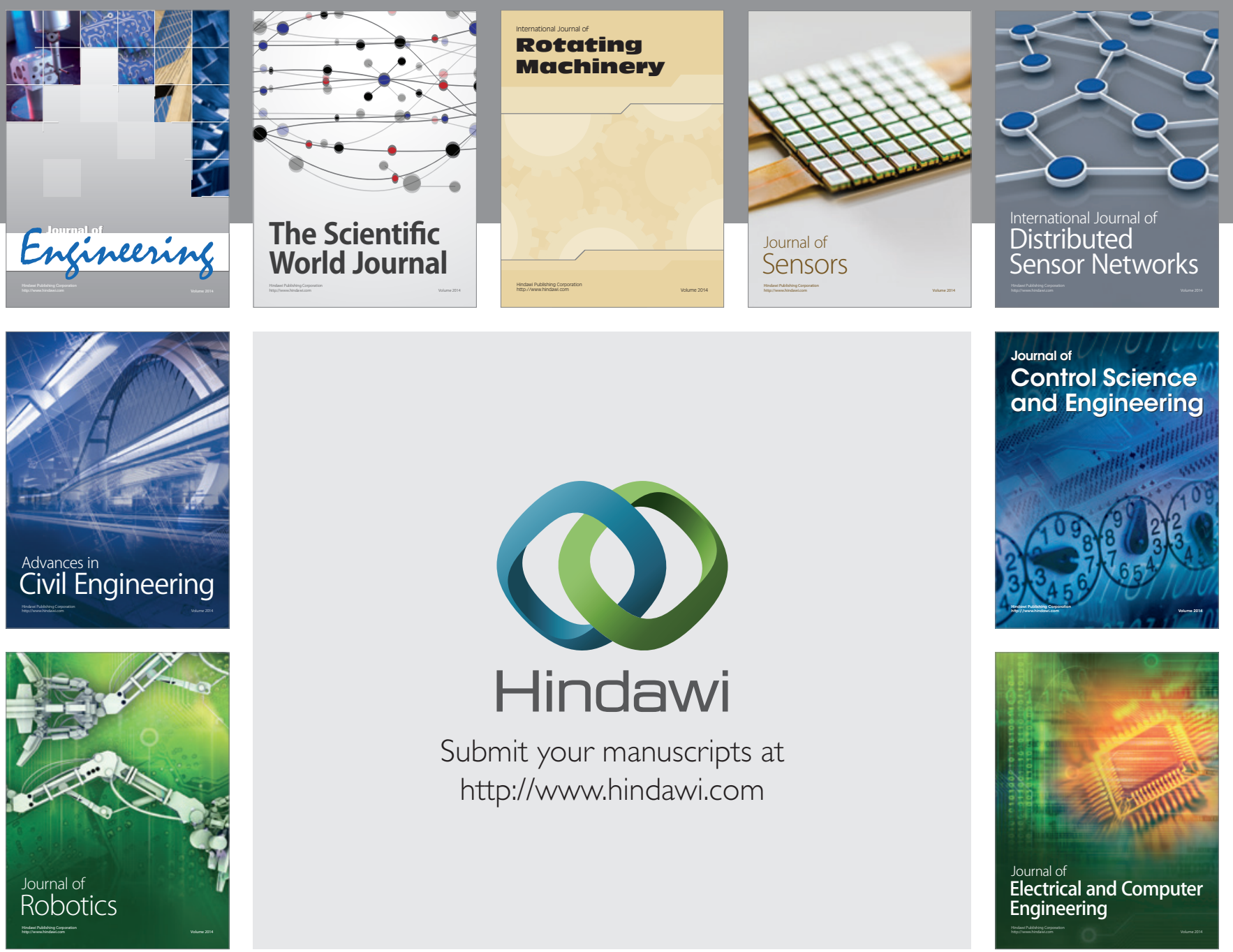

Submit your manuscripts at

http://www.hindawi.com
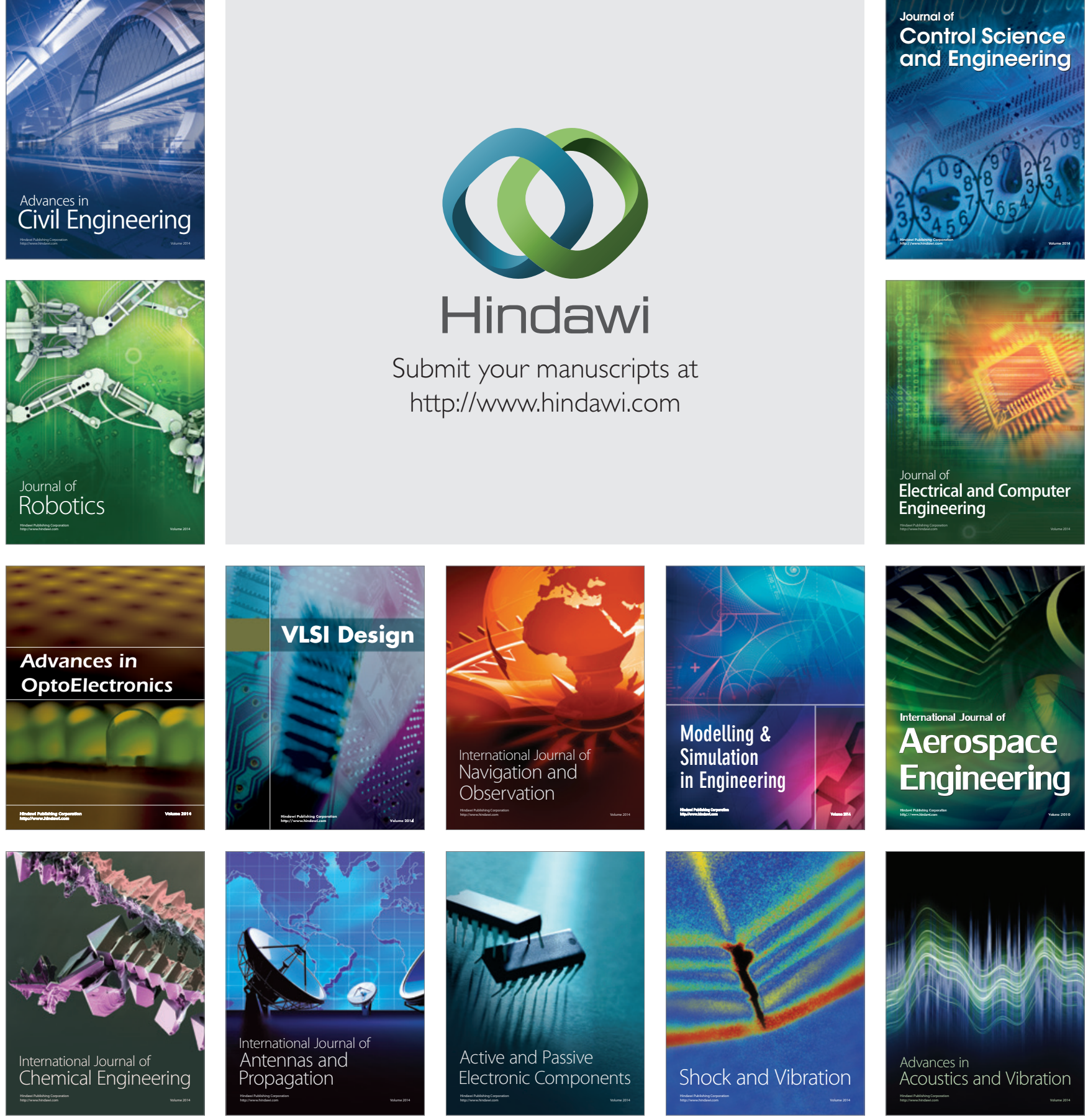